\title{
Electromagnetic/Magnetic-Coupled Targeting System for Screw-Hole Locating in Intramedullary Interlocking-Nail Surgery
}

\author{
Tze-Hong Wong, Tien-Kan Chung, Tzu-Wei Liu, Hou-Jen Chu, Wensyang Hsu, Po-Chen Yeh, \\ Chin-Chung Chen, Meng-Shiue Lee, and Yuh-Shyong Yang
}

\begin{abstract}
At present, intramedullary interlocking nails are widely used for bone-fracture fixation in orthopedic surgeries. Surgeons often use $\mathrm{X}$-ray imaging to find the actual location of the distal screw-holes of the nail after the nail is inserted into the medullary canal of a bone for fixation. Thus, the patients and medical team are inevitably exposed to radioactivity. In this paper, we report a radiation-free electromagnetic/magneticcoupled targeting system to locate the distal screw-holes of the nail used in interlocking-nail surgery. The targeting system consists of a c-shaped electromagnet with a pick-up coil, a highly permeable curved silicon-steel strip embedded on the nail, a guiding mechanism, and electronic measuring instruments. An alternative current is applied to the electromagnet to generate a uniform magnetic field/flux in the electromagnet's air gap. When the nail inserted into the medullary canal of a bone is scanned through or rotated in the air gap of the electromagnet, the magnetic flux in the air gap is influenced by the silicon-steel strip embedded on the nail. The variation of the magnetic flux induces a voltage response in the pick-up coil due to electromagnetic induction. The pattern of the voltage response is analyzed to establish a criterion for screw-hole targeting. The results obtained using this criterion reveal that the maximum targeting error of the location and orientation targeting for a screw-hole with a diameter of $5 \mathrm{~mm}$ is $<2 \mathrm{~mm}$ and $10^{\circ}$, respectively. Thus, the system/approach is sufficiently simple and accurate to be used by surgeons in clinical surgery.
\end{abstract}

Index Terms-Interlocking nail, orthopedic surgery, electromagnetic, magnetic, targeting, locating.

Manuscript received April 6, 2014; revised June 18, 2014; accepted June 18, 2014. Date of publication July 1, 2014; date of current version October 23, 2014. This work was supported in part by the National Chiao Tung University, the National Taiwan University Hospital (Hsinchu Branch) under Grant 102W970-HCH102-09, and in part by the Taiwan Ministry of Science and Technology under Grant 102-2221-E-009-034. The associate editor coordinating the review of this paper and approving it for publication was Prof. Aime Lay-Ekuakille. (Corresponding author: Tien-Kan Chung.)

T.-H. Wong is with the Department of Orthopedics, National Taiwan University Hospital Hsinchu Branch, Hsinchu 30059, Taiwan, and also with the Department of Biological Science and Technology, National Chiao Tung University, Hsinchu 30010, Taiwan (e-mail: ortho@hch.gov.tw).

T.-K. Chung, T.-W. Liu, H.-J. Chu, W. Hsu, P.-C. Yeh, C.-C. Chen, and M.-S. Lee are with the Department of Mechanical Engineering, National Chiao Tung University, Hsinchu 30010, Taiwan (e-mail: tkchung@nctu.edu.tw; f890605@gmail.com; houjen.chu@gmail.com; whsu@mail.nctu.edu.tw; scarletleaf203@gmail.com; aew854262@gmail.com; mengshiuelee@ gmail.com).

Y.-S. Yang is with the Department of Biological Science and Technology, National Chiao Tung University, Hsinchu 30010, Taiwan (e-mail: ysyang@faculty.nctu.edu.tw).

Color versions of one or more of the figures in this paper are available online at http://ieeexplore.iee.org.

Digital Object Identifier 10.1109/JSEN.2014.2334139

\section{INTRODUCTION}

$\mathbf{I}$ NTRAMEDULLARY interlocking nails are widely used in orthopedic surgeries, especially for long-bone fracture fixations. Surgeons insert the nail into the medullary canal to fix the fractured bone. However, after the nail is inserted, the nail becomes invisible to the surgeons (i.e., it cannot be observed by the naked eye). Therefore, surgeons have difficulty confirming the actual location of the distal screw holes of the nail. In addition, the fixation procedure is achieved by locating the nail's distal screw holes and using the screws to fix the nail to the bone. However, when the nail is forcibly inserted into the medullary canal of a bone, the nail is distorted due to the resistant force from the bone. Thus, the actual location and orientation of the distal screw holes of the nail become unpredictable. Without the information regarding the location and orientation of the distal screw holes, the surgeons cannot drill the bone to expose the screw holes for further screw fixation. To address these issues (i.e., the invisibility and unpredictability), an X-ray-image targeting method is used to help surgeons target the location and orientation of the screw holes of the nail inserted into a bone [1]. Although X-ray-imaging targeting is a simple and accurate method for screw-hole targeting, the patients and medical team are inevitably exposed to radioactivity. Thus, a radiation-free targeting method is necessary to avoid this radiation exposure.

Several radiation-free targeting methods/systems (such as mechanical [2]-[5], ultrasonic [6], fiber-optic [7], magneticflux-based [8], and magnetic-force-based [9] targeting methods) have recently been proposed to locate the distal screw holes of a nail inserted into a long bone. However, targeting methods using the mechanical, ultrasonic, fiber-optic, and magnetic-flux-based approaches are imprecise, expensive, inaccurate, and complicated, respectively. The magnetic-forcebased targeting method utilizing the magnetic-force interaction between magnets responds slowly and inaccurately during targeting. Thus, researchers are still searching for a better targeting method. More recently, researchers presented a magnetic-sensor targeting method [10], [11] that is inexpensive and accurate compared to the above-mentioned methods. The magnetic-sensor targeting method uses three hall-effect magnetic sensors to detect the magnetic flux generated by a permanent magnet embedded in the screw hole of a nail inside 


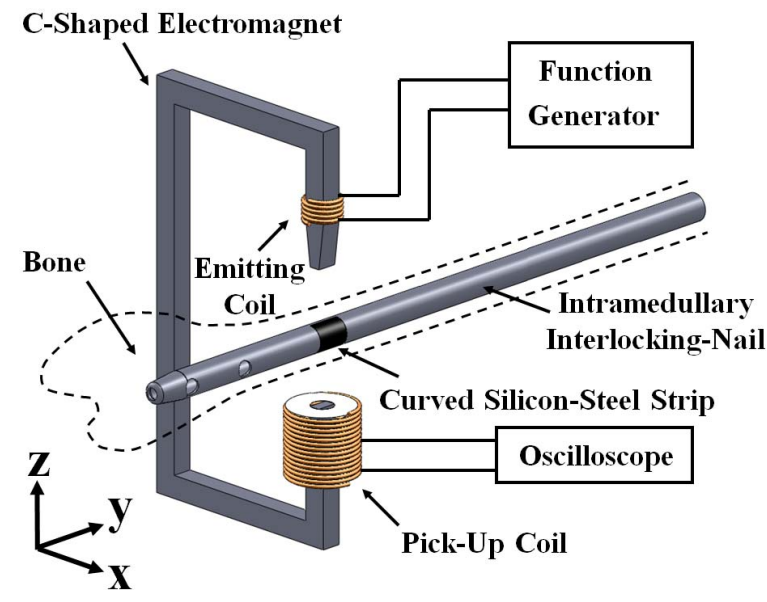

Fig. 1. Targeting approach of the electromagnetic/magnetic-coupled targeting system.

a long bone to predict the location of the screw hole. Although the magnetic-sensor targeting method is inexpensive, accurate, and precise, the targeting algorithm and corresponding circuits for determining the location and orientation of the screw holes are complicated. Therefore, an inexpensive, precise, and accurate yet simple magnetic targeting method is necessary.

To date, many sensing, actuating, transducing, gating, guiding, positioning, and targeting technologies using electromagnetic-induction/magnetic-flux-interactioncoupled approaches have been demonstrated in various applications [12]-[28]. The approaches are inexpensive, precise, accurate, simple, verified, and well developed. Due to these advantages, we believe that these approaches are potential solutions for magnetic targeting during interlockingnail surgery. Hence, in this paper, we present a novel targeting system coupling the electromagnetic induction and magnetic-flux interaction to target the distal screw holes of an interlocking nail inserted into the medullary canal of a long bone.

\section{DESIGN}

The design of the targeting system is illustrated in Figure 1. This design includes a highly permeable curved silicon-steel strip embedded on an intramedullary-interlocking nail and a c-shaped electromagnet with a pick-up coil. The highly permeable curved silicon-steel strip capable of concentrating the magnetic flux is embedded onto the location with a known distance and orientation to the distal screw-holes of nail. After embedding, the nail is inserted into a long bone. An alternative current is applied to the c-shaped electromagnet to generate an alternative magnetic field/flux in the air gap of the electromagnet. When the electromagnet scans through the location with and without the silicon-steel strip embedded on the nail inserted into the medullary canal of the long bone (note: the scanning direction is along the axial direction of the nail, as shown in Figure 1), the magnetic flux is concentrated and non-concentrated, as shown in Figures 2(a) and 2(b), respectively. Thus, the pick-up coil detects different amounts of the flux. A different voltage is induced in the pick-up coil
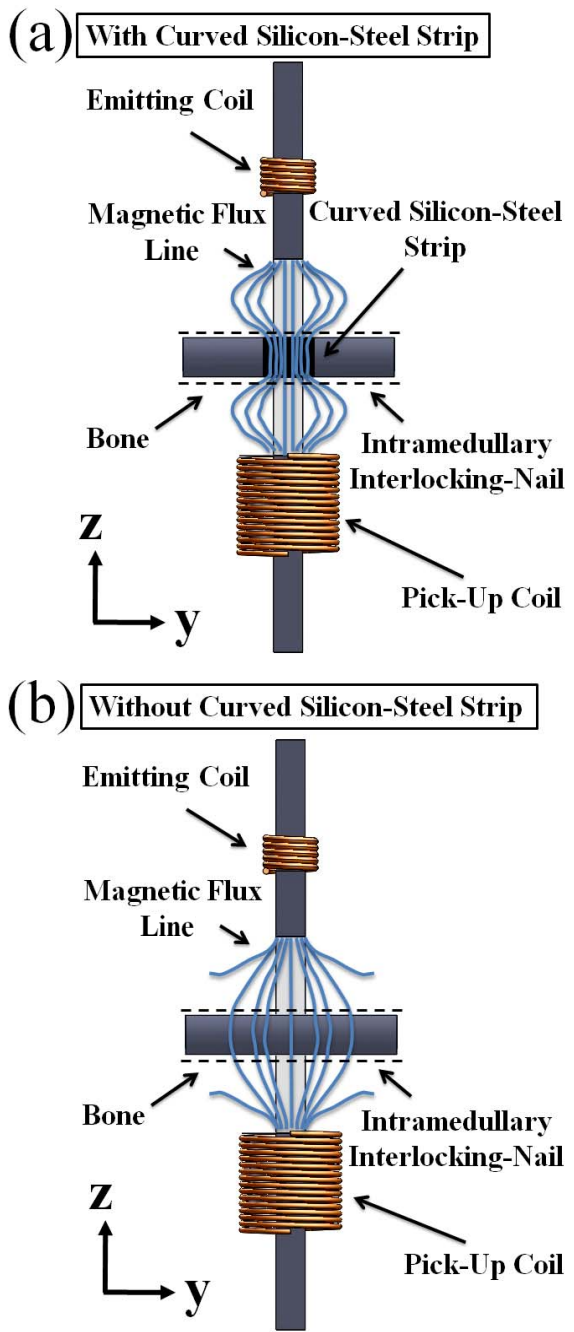

Fig. 2. Location targeting of the distal screw holes of the nail in the bone. (a) Targeted. (b) Not targeted.

due to the electromagnetic induction. The induced voltages are analyzed to predict the location of the silicon-steel strip. The location of the screw holes can be obtained because the relative location between the silicon-steel strip and screw holes is known when the strip is embedded in the nail.

After the location of the distal screw hole is identified, the nail is rotated in the air gap of the electromagnet for orientation targeting, as shown in Figure 3. The magnetic flux generated by the electromagnet is partially concentrated by the silicon-steel strip when the nail is rotated clockwise to an orientation at which the curved silicon-steel strip is perpendicular (i.e., the orientation is defined as 0 degrees) to the c-shaped electromagnet, as shown in Figure 3(a). In contrast, the magnetic flux generated by the electromagnet is mostly concentrated by the silicon-steel strip when the nail is rotated clockwise to an orientation at which the siliconsteel strip is parallel (i.e., the orientation is defined as $90^{\circ}$ ) to the electromagnet, as shown in Figure 3(b). Different voltage responses are induced in the pick-up coil based on the various amounts of the concentrated magnetic flux. The induced voltages are analyzed to predict the location of the silicon-steel strip. The actual orientation of the screw hole 


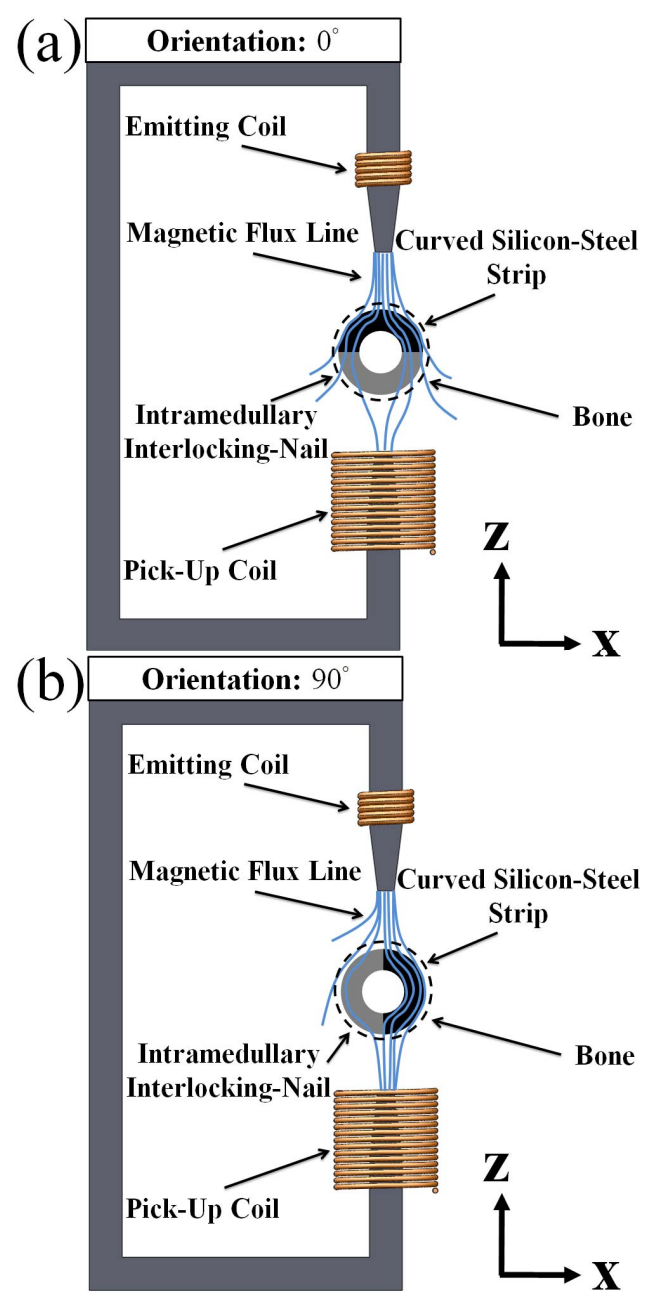

Fig. 3. Orientation targeting of the distal screw holes of the nail in the long bone. (a) 0-degree orientation-targeting. (b) 90-degree orientation targeting.

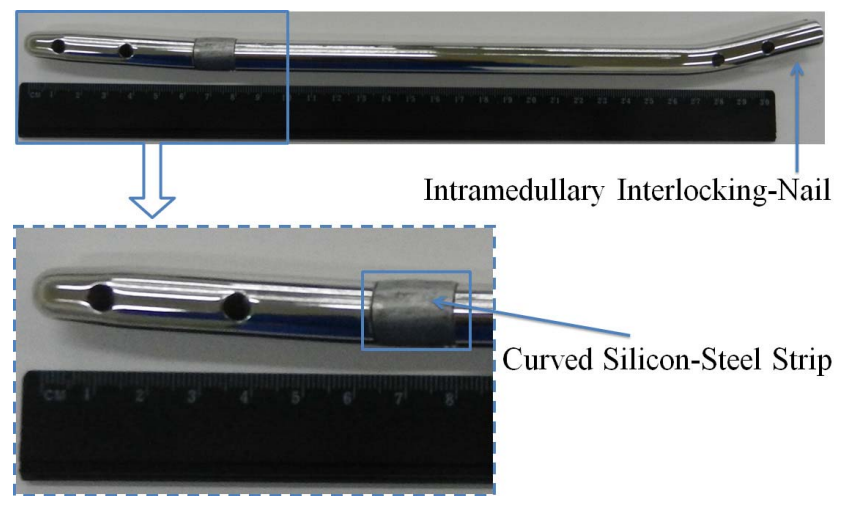

Fig. 4. Photograph of the intramedullary interlocking nail with the embedded highly permeable curved silicon-steel strip.

can be determined because the relative orientation between the silicon-steel strip and screw hole is known when the strip is embedded onto the nail.

\section{FABRICATION}

Based on the design of the targeting system, the system fabrication is divided into four parts: (I) a high-permeable

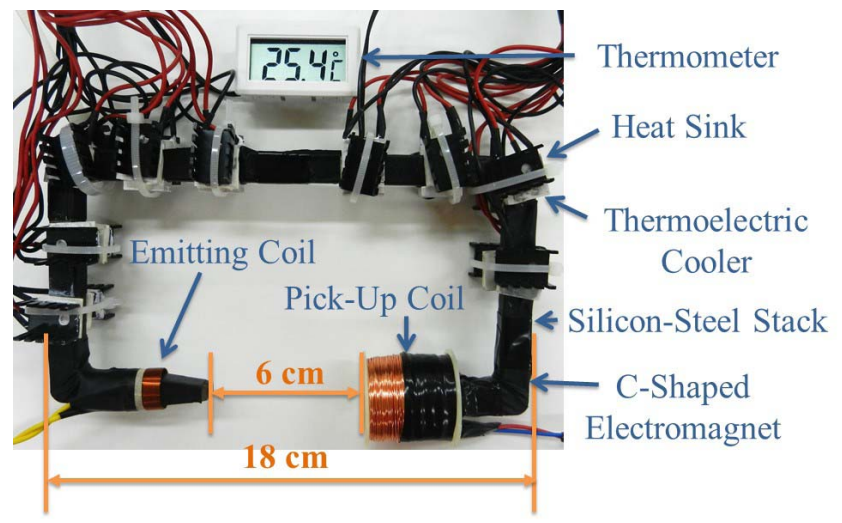

Fig. 5. Photograph of the c-shaped electromagnet with the pick-up coil.

curved silicon-steel strip embedded onto a stainless-steel-based intramedullary interlocking nail, (II) a c-shaped electromagnet with a pick-up coil [8], (III) a guiding mechanism, and (IV) measurement electronics. For the first part, the curved siliconsteel strip fixed on the fabricated nail is shown in Figure 4. A strip region of the nail (R-T Tibial Interlocking-Nail 12-2206, Smith \& Nephew, Inc., Memphis, TN, USA; diameter $\times$ length: $14 \mathrm{~mm} \times 315 \mathrm{~mm}$ ) is removed by machining. Then, the machined strip region is filled and fixed by a highly permeable silicon-steel strip. In this manner, the strip is embedded into the nail.

For the second part, the c-shaped electromagnet with the fabricated pick-up coil is shown in Figure 5. The c-shaped electromagnet consists of a silicon-steel stack, an emitting coil, a thermometer, thermoelectric coolers, and heat sinks. The silicon-steel stack (length $\times$ width $\times$ height $\times$ thickness: $18 \mathrm{~cm} \times 1 \mathrm{~cm} \times 9.5 \mathrm{~cm} \times 0.9 \mathrm{~cm})$ is fabricated by laminating c-shaped silicon-steel sheets. After lamination, 76 turns of enameled wire $(0.15 \mathrm{~mm}$ in diameter $)$ is winded to the silicon-steel stack as the emitting coil. Thermoelectric coolers, heat sinks, and a thermometer are assembled and attached to the silicon-steel stack to form the $\mathrm{c}$-shaped electromagnet. Finally, the pick-up coil (outer diameter $\times$ inner diameter $\times$ thickness: $31.6 \mathrm{~mm} \times 17.14 \mathrm{~mm} \times$ $31 \mathrm{~mm}$ ) consisting of 2,700 turns of winded enameled-wire $(0.286 \mathrm{~mm}$ in diameter) is attached to the c-shaped electromagnet. For the third part, the fabricated guiding mechanism is shown in Figure 6. We assembled the commercial linear and rotary stages on the optical bench as the guiding mechanism. Regarding the last part, the measurement electronics we used include a function generator and oscilloscope.

Finally, the four parts are assembled as the targeting system shown in Figure 7 (excluding the bone in Figure 7).

\section{TESTING}

\section{A. Material Characterization}

To verify whether the curved silicon-steel strip under consideration is highly permeable for our design, the permeability of the strip is estimated through a magnetic hysteresis loop measured by a Superconducting Quantum Interference Device (SQUID). 


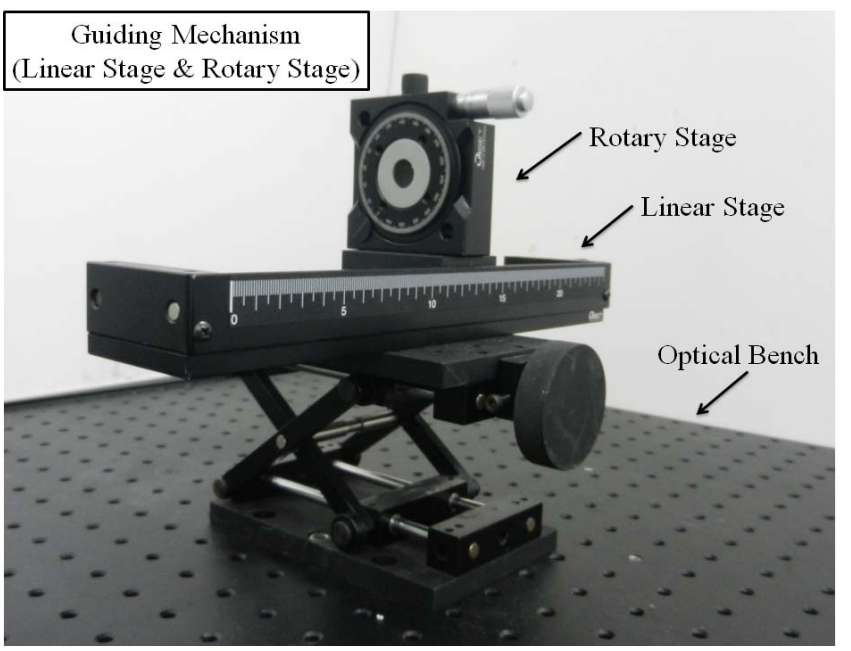

Fig. 6. Photograph of the guiding mechanism.

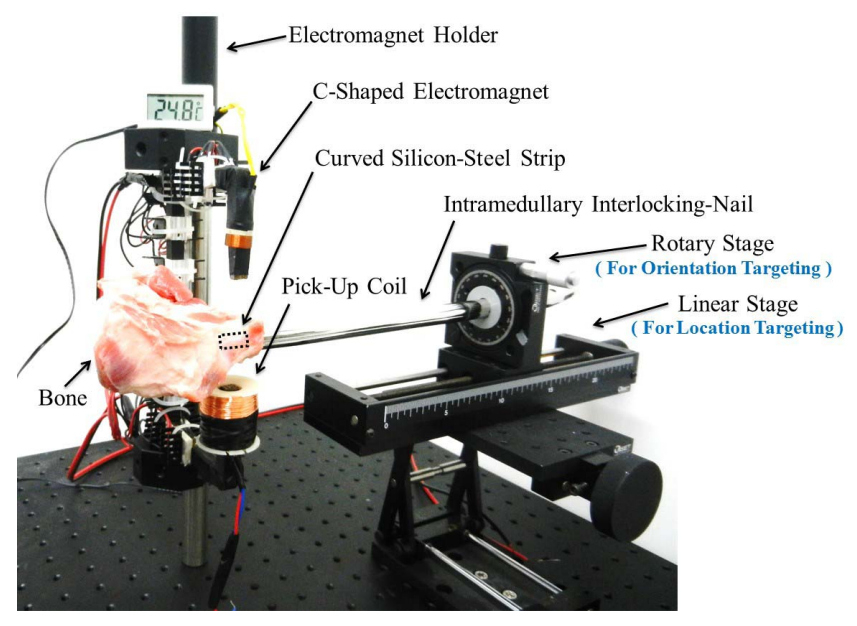

Fig. 7. Photograph of the experimental setup for location- and orientationtargeting tests.

\section{B. Experimental Setup}

Figure 7 displays the experimental setup of the location targeting of the distal screw holes of an intramedullary interlocking nail in a bone. As shown in Figure 7, the distal end of the nail is inserted into a pork bone. The near end of the nail is clamped onto the linear stage of the guiding mechanism. The nail is able to translate through the air gap of the c-shaped electromagnet due to the linear stage of the guiding mechanism. This testing setup allows for the determination of the screw hole locations along the axial direction of the nail. The experimental setup used to determine the orientation of the screw holes is also shown in Figure 7. The rotary stage shown in Figure 7 is used to rotate the nail and thereby rotate the highly permeable curved silicon-steel strip for the orientation measurement. This testing setup allows the orientation of the screw holes to be determined. In both the location and orientation targeting processes, a function generator is used to provide a sinusoidal-wave voltage signal (amplitude: $10.96 \mathrm{~V}$, frequency: $55 \mathrm{kHz}$ ) to the emitting coil of the c-shaped electromagnet to generate an alternative magnetic field/flux in the air gap of the electromagnet. The induced voltage response in the pick-up coil is recorded by an oscilloscope [sampling frequency: $10 \mathrm{MS} / \mathrm{s}$ (mega samples per second), number of bits of the digital converter: 8 bits]. The recorded voltage raw data are consequently exported from the oscilloscope to a computer. The Matlab software is used to obtain the root-mean-square voltage from the voltage raw data.

\section{Targeting and Analyzing Procedure}

The targeting and corresponding analysis is a two-step procedure. The first step is to set location and orientation reference points. The second step is to scan the bone. The details of the two steps are described below.

(I) Setting Reference Points:

To verify whether we can successfully target the screw hole of the nail inside a bone, we use a pig leg bone instead of a real human leg bone in our experiment. The pig leg bone is considerably shorter than the human leg bone. In addition, the diameter of the medullary canal of the pig leg bone is slightly larger than that of the nail. Due to the shorter length and larger diameter of the pig leg bone, the bone produces no resistance to the nail when the nail is inserted into the medullary canal of the bone [note: however, during clinical surgery, the real human's leg bone (i.e., long bone) produces a large resistance to the nail due to the geometric discrepancy (length and diameter) between the long bone and nail]. For the pig leg bone (i.e., short bone) used in our experiment, the nail undergoes no deformation and distortion while being inserted into the bone. Thus, after the nail is inserted into the short bone, we can determine the actual location and orientation of the screw hole/silicon-steel stripe of the nail by marking the location and orientation reference points on the linear and rotatory stage, respectively.

(II) Scanning the Bone:

We perform the first location scan after the location and orientation reference points are set on the linear and rotatory stages. We use the c-shape electromagnet to scan the bone (nail inside) from left to right along the axial direction of the nail, as illustrated in Figure 10(b), i.e., from point A to point $\mathrm{E}$ in Figure 10(b). In the scan, the incremental step is $2 \mathrm{~mm}$. In each step, we use an oscilloscope to record the induced voltage output. The recorded voltage output is exported from the oscilloscope to a computer. The rootmean-square voltage $\left(\mathrm{V}_{\mathrm{rms}}\right)$ of the voltage output is obtained using Matlab. Next, we perform a second location scan with the same scanning parameters, but the scanning direction is from right to left, i.e., from point $\mathrm{E}$ to point $\mathrm{A}$. We also obtain the corresponding $\mathrm{V}_{\mathrm{rms}}$ in the second scan. Finally, the $\mathrm{V}_{\mathrm{rms}}$ of the two scans are averaged (as $\mathrm{V}_{\text {rms, two-scans). }}$ ). Furthermore, we perform 10 experimental runs, where each repetition includes the above-mentioned two scans. Based on the 10 runs, we are able to plot a curve that illustrates the averaged voltage response $\left(\mathrm{V}_{\mathrm{rms}}\right.$, averaged $)$ over the 10 runs at the different scanned locations. The location-targeting error is estimated by comparing the actual location of the silicon-steel strip embedded into the nail inserted into the bone (i.e., the location reference point) and the predicted location represented by the maximum $\mathrm{V}_{\mathrm{rms}}$, averaged 


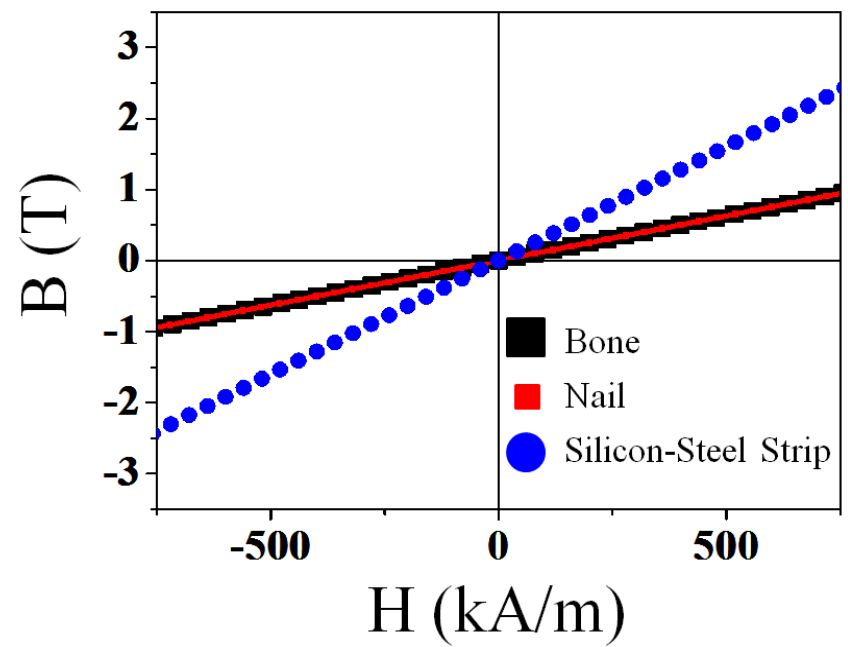

Fig. 8. Magnetic hysteresis loop of the bone (black curve), nail (red curve) and silicon-steel strip (blue curve).

We perform the first orientation scan after the location is targeted. We use the c-shape electromagnet to scan the bone (with the nail inside), which is rotated by a rotary stage from $0^{\circ}$ to $180^{\circ}$, as illustrated in Figure $12(\mathrm{~b})$. In the scan, the incremental step is $5^{\circ}$. In each step, we use an oscilloscope to record the induced voltage output. The recorded voltage output is exported from the oscilloscope to a computer. The rootmean-square voltage $\left(\mathrm{V}_{\mathrm{rms}}\right)$ of the voltage output is obtained using Matlab. Next, we perform a second orientation scan with the same scanning parameters, but the scanning direction is from $180^{\circ}$ to $0^{\circ}$. We also obtain the corresponding $\mathrm{V}_{\mathrm{rms}}$ in the second scan. Finally, the $V_{\text {rms }}$ of the two scans are averaged (as $\mathrm{V}_{\text {rms, two-scans) }}$ ). Furthermore, we perform 10 experimental runs, with each repetition including the above-mentioned two scans. Based on the 10 runs, we are able to plot a curve that illustrates the averaged voltage response $\left(\mathrm{V}_{\text {rms, averaged }}\right)$ over the 10 runs at the different scanned orientations. The orientation-targeting error is estimated by comparing the actual orientation of the silicon-steel strip embedded into the nail inserted into the bone (i.e., the orientation reference point) and the predicted orientation represented by the maximum $\mathrm{V}_{\text {rms, averaged. }}$

\section{Results AND Discussion}

The magnetic hysteresis loops of the bone, nail, and curved silicon-steel strip measured by SQUID are shown in Figure 8. The relative permeabilities of the bone, nail, and silicon-steel strip are $0.99,1$, and 44.6 , respectively (note: the relative permeability of the air is 1). Thus, the silicon-steel strip considered here has a relatively high permeability that is capable of concentrating the magnetic flux compared to the bone and nail.

The experimental results of the location targeting of the distal screw holes along the axial direction of the nail are presented in Figures 9 and 10 and Table 1. Figure 9 presents the raw induced voltage responses (root-mean-square voltage, $V_{\text {rms }}$ ) at different scanned locations (note: the scan is performed along the axial direction of the nail from the distal to central section of the nail) according to 10 experimental

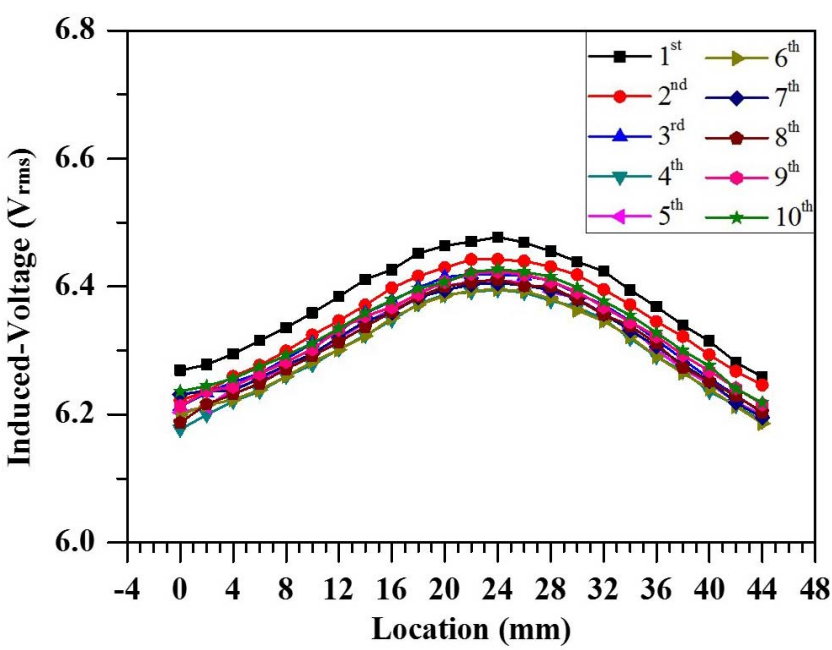

Fig. 9. The experimental raw results of the location targeting obtained from 10 experimental runs.

TABLE I

Averaged Induced RoOt-MeAn-SQuare Voltage ( $\mathrm{V}_{\text {RMS }}$ ) AT DIFFERENT SCANNED LOCATIONS BASED ON TEN EXPERIMENTAL RUNS

\begin{tabular}{|c|c|c|c|c|c|}
\hline \multirow{2}{*}{$\begin{array}{l}\text { Location } \\
\quad(\mathrm{mm})\end{array}$} & \multicolumn{2}{|c|}{ Induced Voltage $\left(\mathrm{V}_{\mathrm{rms}}\right)$} & \multirow{2}{*}{$\begin{array}{l}\text { Location } \\
(\mathrm{mm})\end{array}$} & \multicolumn{2}{|c|}{ Induced Voltage $\left(\mathrm{V}_{\mathrm{rms}}\right)$} \\
\hline & Mean & $\begin{array}{l}\text { Standard } \\
\text { Deviation }\end{array}$ & & Mean & $\begin{array}{l}\text { Standard } \\
\text { Deviation }\end{array}$ \\
\hline 0 & 6.215 & 0.026 & $24 *$ & $6.421 *$ & $0.025 *$ \\
\hline 2 & 6.231 & 0.022 & 26 & 6.416 & 0.024 \\
\hline 4 & 6.246 & 0.022 & 28 & 6.406 & 0.023 \\
\hline 6 & 6.263 & 0.023 & 30 & 6.391 & 0.023 \\
\hline 8 & 6.284 & 0.022 & 32 & 6.370 & 0.024 \\
\hline 10 & 6.305 & 0.024 & 34 & 6.346 & 0.023 \\
\hline 12 & 6.329 & 0.024 & 36 & 6.318 & 0.025 \\
\hline 14 & 6.353 & 0.026 & 38 & 6.289 & 0.025 \\
\hline 16 & 6.373 & 0.024 & 40 & 6.264 & 0.025 \\
\hline 18 & 6.394 & 0.024 & 42 & 6.235 & 0.023 \\
\hline 20 & 6.409 & 0.023 & 44 & 6.211 & 0.024 \\
\hline 22 & 6.418 & 0.024 & & & \\
\hline
\end{tabular}

*The repeatability in the location of the maximum induced voltage (over the 10 runs) is $80 \%$, which is calculated from the following equation: [(percentage of cases in which the maximum voltage corresponds to the location 24 $\mathrm{mm}) / 10]^{*} 100$

runs. The raw voltage data from Figure 9 are averaged in Table 1 and shown in Figure 10(a).

The experimental mean value and standard deviation of the induced root-mean-square voltage at each location in Figures 9 and 10(a) and Table 1 indicate that the system/approach has good repeatability. Furthermore, when we compared the predicted location scanned by the electromagnet (i.e., the scanned location from points A to E marked in Figure 10(a)) and the actual location of the curved silicon-steel strip indicated by the location reference point on the stage (i.e., correlated points A to E illustrated in Figure 10(b)), the induced voltage response reaches the maximum $(6.42 \mathrm{~V}$, location: $24 \mathrm{~mm})$ while scanning around the central section of the silicon-steel strip embedded in the nail, as indicated by point $\mathrm{C}$ (location: $22 \mathrm{~mm}$ ) in Figures 10(a) and 10(b). In addition, while scanning at the location with and without the silicon-steel strip, the maximum variation of the voltage response is $0.21 \mathrm{~V}$, which 

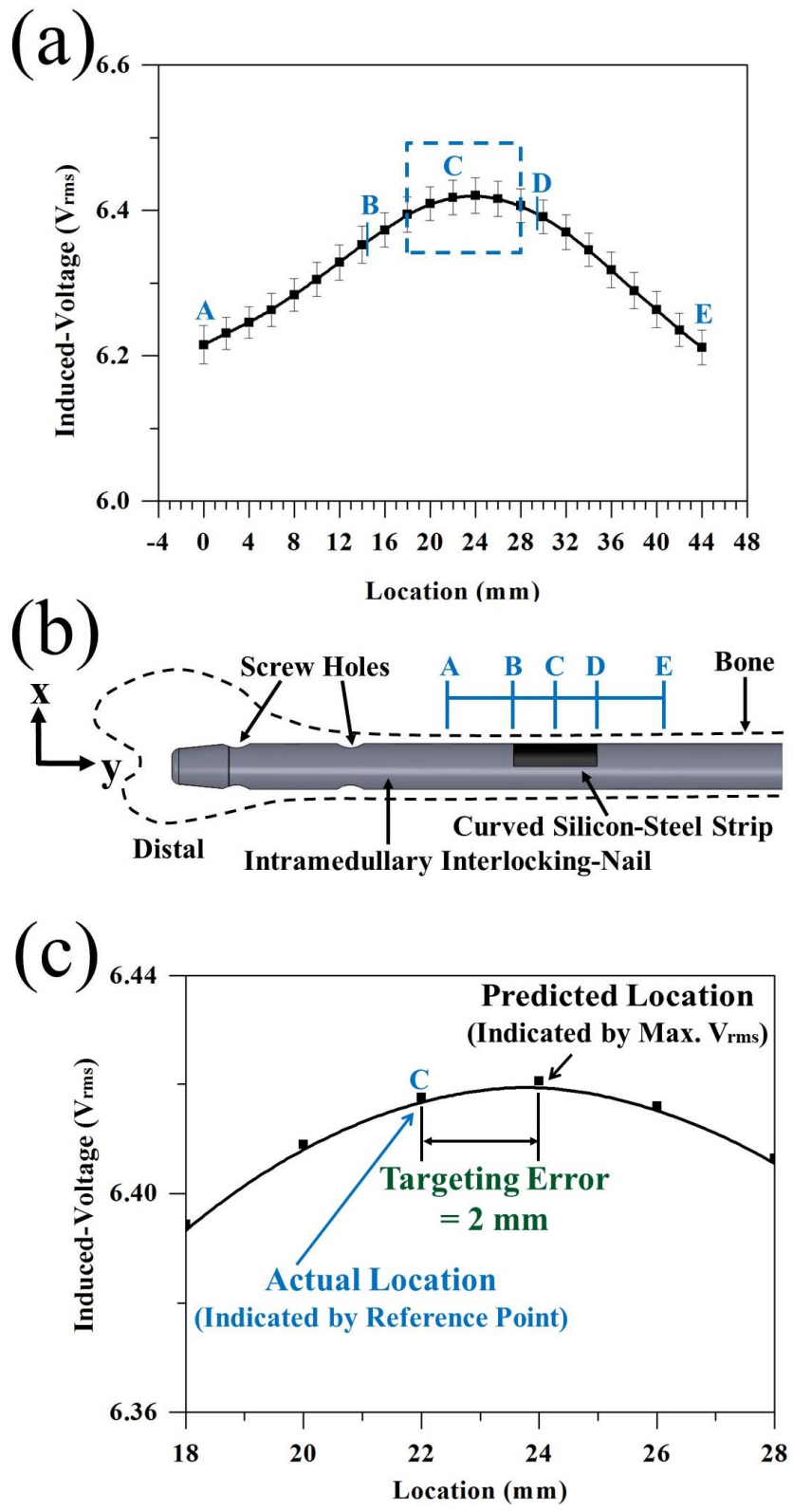

Fig. 10. Experimental result of the location targeting: (a) The averaged induced-root-mean-square voltage at different scanned locations. (b) The actual/reference location is correlated with the scanned location. (c) The enlarged voltage pattern from the dash-line boxed region in (a).

is clearly distinguishable from the noise. Moreover, the voltage pattern in Figure 10(c), which is enlarged from Figure 10(a), can estimate the location targeting's experimental error. The targeting error is $2 \mathrm{~mm}$ which is estimated by comparing the prediction location (at $24 \mathrm{~mm}$ ) indicated by the maximum voltage response and actual location (at $22 \mathrm{~mm}$ ) of the siliconsteel strip indicated by the reference point on the stage. In addition, the repeatability in the prediction location of the maximum induced voltage (over the 10 runs) is $80 \%$, which is calculated from the following equation: [(percentage of cases in which the maximum voltage corresponds to the location $24 \mathrm{~mm}) / 10]^{*} 100$. These experimental data demonstrate the validity of the location-targeting approach (that is, the maximum induced voltage response predicts the actual location of

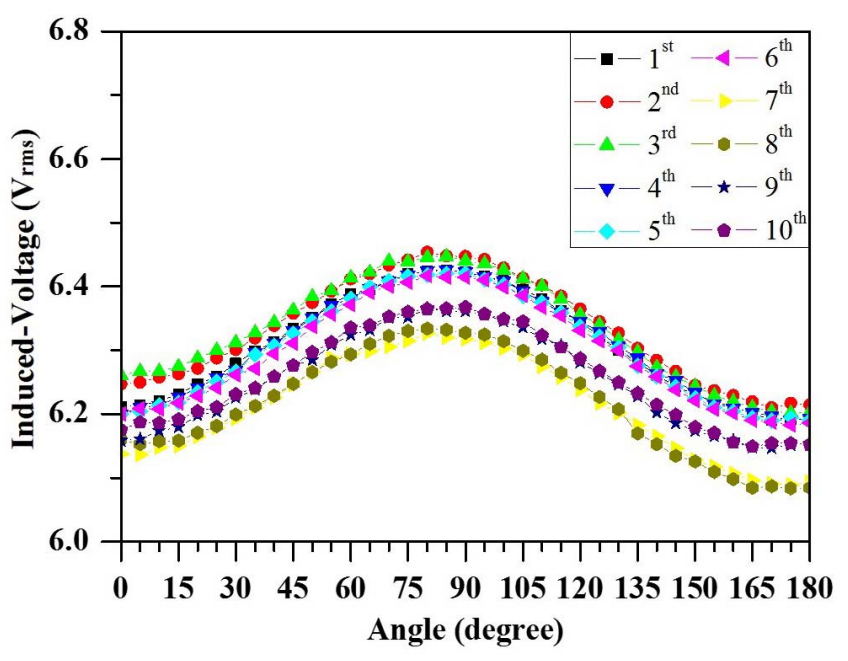

Fig. 11. The experimental raw results of the orientation targeting obtained from ten experimental runs.

TABLE II

AVERAGEd INDUCED-RoOT-MEAN-SQuare Voltage ( $\left.\mathrm{V}_{\mathrm{RMS}}\right)$ AT DIFFERENT SCANNED ORIENTATIONS BASED ON TEN EXPERIMENTAL RUNS

\begin{tabular}{|c|c|c|c|c|c|}
\hline \multirow{2}{*}{$\begin{array}{c}\text { Angle } \\
\text { (degree) }\end{array}$} & \multicolumn{2}{|c|}{ Induced Voltage $\left(\mathrm{V}_{\mathrm{rms}}\right)$} & \multirow{2}{*}{$\begin{array}{l}\text { Angle } \\
\text { (degree) }\end{array}$} & \multicolumn{2}{|c|}{ Induced Voltage $\left(\mathrm{V}_{\mathrm{rms}}\right)$} \\
\hline & Mean & $\begin{array}{c}\text { Standard } \\
\text { Deviation }\end{array}$ & & Mean & $\begin{array}{l}\text { Standard } \\
\text { Deviation }\end{array}$ \\
\hline 0 & 6.195 & 0.039 & 95 & 6.388 & 0.047 \\
\hline 5 & 6.199 & 0.041 & 100 & 6.379 & 0.046 \\
\hline 10 & 6.204 & 0.039 & 105 & 6.366 & 0.044 \\
\hline 15 & 6.211 & 0.041 & 110 & 6.350 & 0.047 \\
\hline 20 & 6.225 & 0.041 & 115 & 6.333 & 0.046 \\
\hline 25 & 6.237 & 0.042 & 120 & 6.314 & 0.046 \\
\hline 30 & 6.254 & 0.040 & 125 & 6.295 & 0.047 \\
\hline 35 & 6.271 & 0.043 & 130 & 6.276 & 0.046 \\
\hline 40 & 6.288 & 0.043 & 135 & 6.253 & 0.048 \\
\hline 45 & 6.307 & 0.043 & 140 & 6.233 & 0.047 \\
\hline 50 & 6.326 & 0.044 & 145 & 6.217 & 0.048 \\
\hline 55 & 6.344 & 0.042 & 150 & 6.201 & 0.046 \\
\hline 60 & 6.360 & 0.045 & 155 & 6.188 & 0.046 \\
\hline 65 & 6.370 & 0.046 & 160 & 6.179 & 0.047 \\
\hline 70 & 6.383 & 0.047 & 165 & 6.169 & 0.048 \\
\hline 75 & 6.390 & 0.046 & 170 & 6.166 & 0.045 \\
\hline $80 *$ & $6.398 *$ & $0.046^{*}$ & 175 & 6.165 & 0.046 \\
\hline 85 & 6.396 & 0.047 & 180 & 6.166 & 0.045 \\
\hline 90 & 6.394 & 0.047 & & & \\
\hline
\end{tabular}

*The repeatability in the orientation of the maximum induced voltage (over the 10 runs) is $50 \%$, which is calculated from the following equation: [(percentage of cases in which the maximum voltage corresponds to the orientation $\left.\left.80^{\circ}\right) / 10\right]^{*} 100$.

the silicon-steel strip). After the actual location of the strip is determined, the location of the screw hole is determined from the known distance between the strip and screw hole. Finally, surgeons can easily and accurately locate the screw hole of the nail in the bone through the targeting approach by comparing the targeting error $(2 \mathrm{~mm})$ with the diameter of the screw hole (5 mm).

After the location of the silicon-steel strip is determined, the orientation of the strip is determined by the orientationtargeting method. The experimental result of the orientation 

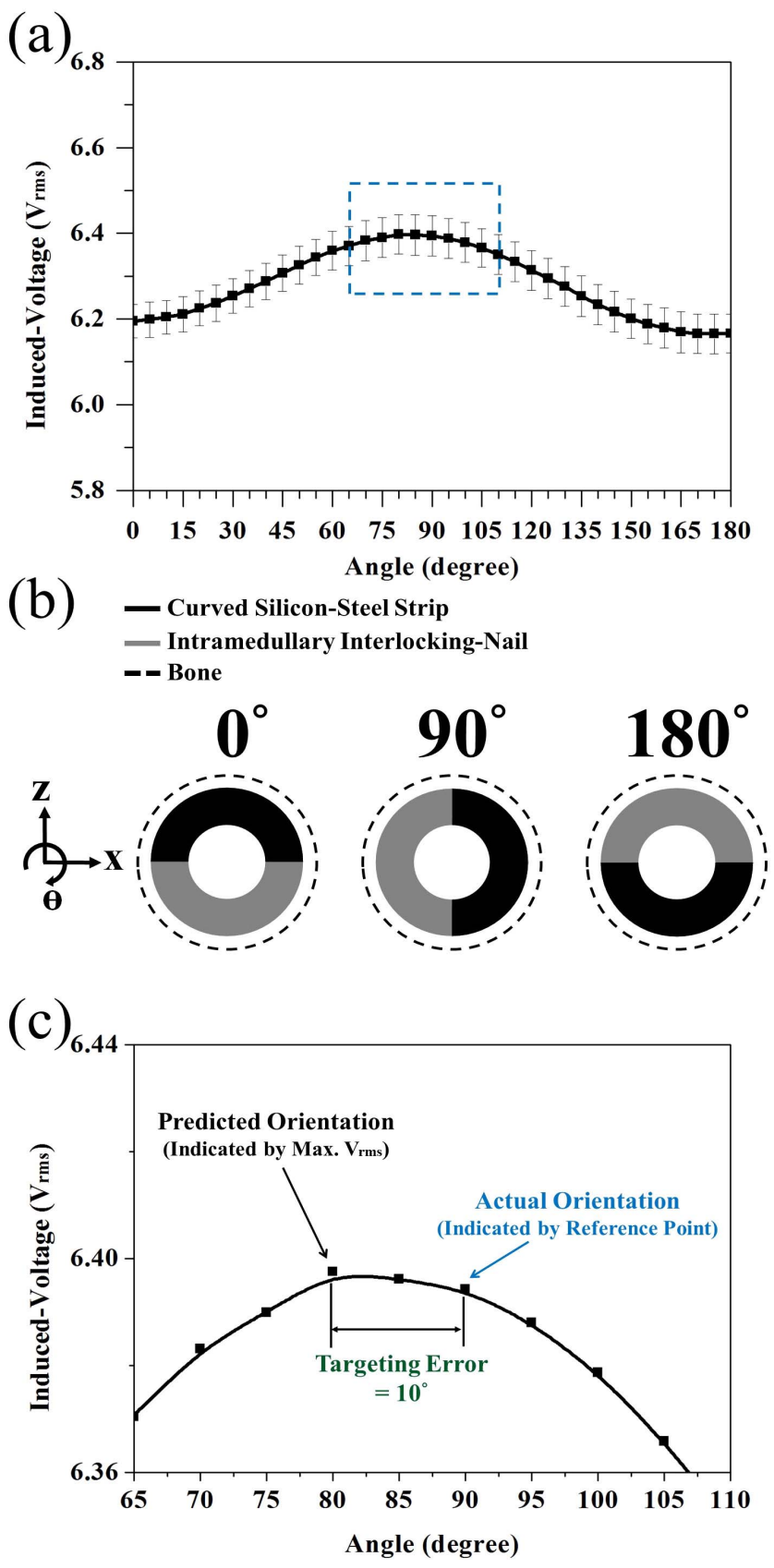

Fig. 12. Experimental result of the orientation targeting: (a) The averaged induced-root-mean-square voltage at different scanned orientations. (b) The actual/reference orientation angle is correlated with the scanned orientation angle. (c) The enlarged voltage pattern from the dash-line boxed region in (a).

targeting of the strip (the nail rotates in the air gap of the electromagnet, as presented in Figure 3) is shown in Figures 11 and 12 and Table 2. As shown in Figure 11, the raw induced voltage response (root-mean-square voltage, $\mathrm{V}_{\mathrm{rms}}$ ) at different scanned orientations (scanned clockwise from $0^{\circ}$ to $180^{\circ}$, as shown in Figure 3) is recorded in each of the 10 experimental runs. The raw voltage data from Figure 11 are averaged in Table 2 and shown in Figure 12(a).

As shown in Figures 11 and 12(a) and Table 2, the experimental mean value and standard deviation of the induced root-mean-square voltage at each orientation indicate that

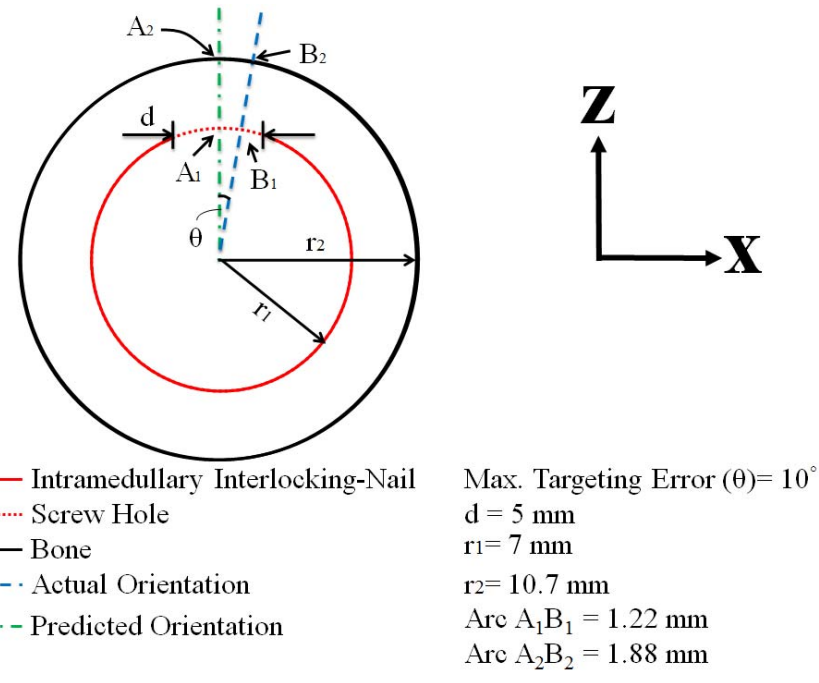

Fig. 13. Cross-sectional geometry-configured estimation for correlating the orientation targeting error in degree to the equivalent location targeting error in length when targeting the 5-mm-diameter screw hole.

the system/approach has a good repeatability. Furthermore, when comparing the predicted orientation scanned by the electromagnet [i.e., the orientation angle in Figure 12(a)] and the actual orientation of the silicon-steel strip indicated by the orientation reference point on the stage [i.e., illustrated in Figure 12(b)], the induced voltage response reaches the maximum $\left(6.40 \mathrm{~V}\right.$, angle: $\left.80^{\circ}\right)$ while scanning at approximately $90^{\circ}$. While scanning at $90^{\circ}$ and $0^{\circ}$ (or $90^{\circ}$ and $180^{\circ}$ ), the maximum variation of the voltage response is approximately $0.23 \mathrm{~V}$, which is clearly distinguishable from the noise. Moreover, the voltage pattern in Figure 12(c), which is enlarged from Figure 12(a), can estimate the orientation-targeting experimental error. The targeting error is $10^{\circ}$ which is estimated by comparing the prediction orientation $\left(\right.$ at $80^{\circ}$ ) indicated by the maximum voltage response and actual orientation (at $90^{\circ}$ ) of the silicon-steel strip indicated by the reference point on the stage. In addition, the repeatability in the orientation of the maximum induced voltage (over the 10 runs) is 50\%, which is calculated from the following equation: [(percentage of cases in which the maximum voltage corresponds to the $80^{\circ}$ orientation $\left./ 10\right]^{*} 100$. When we consider orientation range in repeatability calculation, the repeatability in the $80^{\circ} \pm 5^{\circ}$ orientation range of the maximum induced voltage (over the 10 runs) is up to $80 \%$. These experimental evidences validate the orientation-targeting approach, i.e., the maximum induced voltage is clearly capable of predicting the actual orientation of the silicon-steel strip. After the actual orientation of the strip is determined, the orientation of the screw hole is determined from the known distance and relative orientation between the strip and screw hole. Furthermore, according to the crosssectional geometric configuration of the 5-mm-diameter screw hole of the nail inserted into the medullary canal of the bone, as shown in Figure 13 (note: we assume that the nail and bone are concentric to simplify the estimation of the equivalent targeting error), the targeting error of $10^{\circ}$ is estimated to be equivalent to the length of the arc $\mathrm{A}_{2} \mathrm{~B}_{2}$, which is $1.88 \mathrm{~mm}$. 
That is, the targeting error of $10^{\circ}$ is equivalent to a targeting error of $1.88 \mathrm{~mm}$ for targeting the 5 -mm-diameter screw hole. Thus, the orientation-targeting approach is sufficiently accurate for use by surgeons during clinical interlocking-nail surgery.

\section{CONCLUSION}

We presented a radiation-free electromagnetic/magneticcoupled targeting system for locating the distal screw holes of an intramedullary interlocking nail during long-bone fracture surgery. When targeting a distal screw hole with a diameter of $5 \mathrm{~mm}$, the experimental results reveal that the maximum targeting error is less than $2 \mathrm{~mm}$ and $10^{\circ}$ in the location and orientation targeting of the screw hole, respectively. Therefore, surgeons can easily and accurately target the screw hole of the nail within the bone. The approach/system is sufficiently simple and accurate to be used by surgeons during clinical interlocking-nail surgery. In the future, we will conduct clinical animal tests to verify the use of our targeting system for such surgery.

\section{REFERENCES}

[1] G. M. Whatling and L. D. M. Nokes, "Literature review of current techniques for the insertion of distal screws into intramedullary locking nails," Injury, vol. 37, no. 2, pp. 109-119, 2006.

[2] Z. Gugala, A. Nana, and R. W. Lindsey, "Tibial intramedullary nail distal interlocking screw placement: Comparison of the free-hand versus distally-based targeting device techniques," Injury, vol. 32, pp. 21-25, Dec. 2001.

[3] S. Tyropoulos and C. Garnavos, "A new distal targeting device for closed interlocking nailing," Injury, vol. 32, no. 9, pp. 732-735, 2001.

[4] K. M. Abdlslam and F. Bonnaire, "Experimental model for a new distal locking aiming device for solid intramedullary tibia nails," Injury, vol. 34, no. 5, pp. 363-366, 2003.

[5] M.-Y. Lee, C.-H. Kuo, and S.-S. Hung, "Implementation of a sound-guided navigation system for tibial closed interlocking nail fixations in orthopedics," J. Med. Biol. Eng., vol. 24, no. 3, pp. 147-153, 2004

[6] Y. Ikeda, S. Kobashi, K. Kondo, and Y. Hata, "Fuzzy ultrasonic array system for locating screw holes of intramedullary nail," in Proc. IEEE Int. Conf. Syst., Man, Cybern., Oct. 2007, pp. 2929-2933.

[7] W. Chu, J. Wang, S.-T. Young, and W. C. Chu, "Reducing radiation exposure in intra-medullary nailing procedures: Intra-medullary endo-transilluminating (iMET)," Injury, vol. 40, no. 10, pp. 1084-1087, 2009.

[8] T.-K. Chung et al., "An electromagnetic-induction approach for screw-hole targeting in interlocking-nail surgery," in Proc. IEEE Sensors Conf., Oct. 2012, pp. 1-4.

[9] M.-S. Lee, S.-Y. Wu, T.-H. Wong, W. Hsu, and T.-K. Chung, "A novel guiding device for distal locking of intramedullary nails," in Proc. IEEE Sensors Conf., Oct. 2012, pp. 1-4.

[10] M. Rigoberto and M. Arturo, "Assistant system for locking intramedullary nails used to repair fractures of the long bones," in Proc. Int. Conf. Electr. Electron. Eng., Sep. 2007, pp. 65-67.

[11] M. R. Martinez and M. A. Minor, "A system for distal fixation of intramedullary nails," Int. J. Appl. Electromagn. Mech., vol. 32, no. 1, pp. 21-30, 2010.

[12] S. Foong and K.-M. Lee, "Magnetic field-based multi-DOF orientation sensor for PM-based spherical actuators," in Proc. IEEE/ASME Int. Conf. Adv. Intell. Mechatron., Jul. 2009, pp. 481-486.

[13] M. A. Reed and W. R. Scott, "Coil optimization method for electromagnetic induction systems," IEEE Sensors J., vol. 13, no. 11, pp. 4506-4512, Nov. 2013.

[14] X. Sun et al., "Operation-state monitoring and energization-status identification for underground power cables by magnetic field sensing," IEEE Sensors J., vol. 13, no. 11, pp. 4527-4533, Nov. 2013.

[15] M. I. Beyaz, B. Hanrahan, J. Feldman, and R. Ghodssi, "Monitoring of actuation conditions in a micro-turbo-generator," IEEE Sensors J., vol. 13, no. 8, pp. 2937-2943, Aug. 2013.
[16] S. Taghvaeeyan, R. Rajamani, and Z. Sun, "Non-intrusive piston position measurement system using magnetic field measurements," IEEE Sensors J., vol. 13, no. 8, pp. 3106-3114, Aug. 2013.

[17] A. Ajbl, M. Pastre, and M. Kayal, "A fully integrated hall sensor microsystem for contactless current measurement," IEEE Sensors J., vol. 13, no. 6, pp. 2271-2278, Jun. 2013.

[18] J. B. Faria and M. G. Neves, "Induction coil system for 3-D position sensing of an off-centered tilted primary wire," IEEE Sensors J., vol. 12, no. 10, pp. 2923-2930, Oct. 2012.

[19] G. T. Laskoski, S. F. Pichorim, and P. J. Abatti, "Distance measurement with inductive coils," IEEE Sensors J., vol. 12, no. 6, pp. 2237-2242, Jun. 2012

[20] A. Markham, N. Trigoni, D. W. Macdonald, and S. A. Ellwood, "Underground localization in 3-D using magneto-inductive tracking," IEEE Sensors J., vol. 12, no. 6, pp. 1809-1816, Jun. 2012.

[21] A. P. J. van Deursen and V. Stelmashuk, "Inductive sensor for lightning current measurement, fitted in aircraft windows-Part I: Analysis for a circular window," IEEE Sensors J., vol. 11, no. 1, pp. 199-204, Jan. 2011

[22] E. L. Tan, B. D. Pereles, and K. G. Ong, "A wireless embedded sensor based on magnetic higher order harmonic fields: Application to liquid pressure monitoring," IEEE Sensors J., vol. 10, no. 6, pp. 1085-1090, Jun. 2010.

[23] P. Ripka and M. Janosek, "Advances in magnetic field sensors," IEEE Sensors J., vol. 10, no. 6, pp. 1108-1116, Jun. 2010.

[24] C. Hu, M. Li, S. Song, W. Yang, R. Zhang, and M. Q.-H. Meng, "A cubic 3-axis magnetic sensor array for wirelessly tracking magnet position and orientation," IEEE Sensors J., vol. 10, no. 5, pp. 903-913, May 2010.

[25] S.-M. Yang and C.-L. Huang, "A hall sensor-based three-dimensional displacement measurement system for miniature magnetically levitated rotor," IEEE Sensors J., vol. 9, no. 12, pp. 1872-1878, Dec. 2009.

[26] J. Lenz and A. S. Edelstein, "Magnetic sensors and their applications," IEEE Sensors J., vol. 6, no. 3, pp. 631-649, Jun. 2006.

[27] X. Ma, A. J. Peyton, R. Binns, and S. R. Higson, "Electromagnetic techniques for imaging the cross-section distribution of molten steel flow in the continuous casting nozzle," IEEE Sensors J., vol. 5, no. 2, pp. 224-232, Apr. 2005.

[28] H. Sumali, E. P. Bystrom, and G. W. Krutz, "A displacement sensor for nonmetallic hydraulic cylinders," IEEE Sensors J., vol. 3, no. 6, pp. 818-826, Dec. 2003.

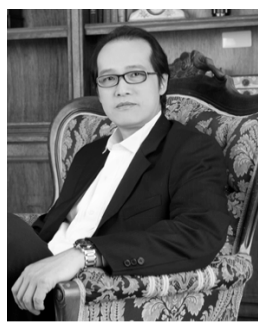

Tze-Hong Wong received the B.Sc. degree in medical technology from National Taiwan University, Taipei, Taiwan, and the M.D. and E.M.B.A. degrees. He has been the Chair of the Department of Orthopedics, National Taiwan University Hospital Hsin Chu, Hsinchu, Taiwan, for ten years. He is most interested in the field of spinal surgery and artsroplasty operation, and new design of medical implants.

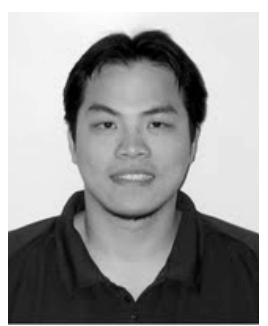

Tien-Kan Chung is an Assistant Professor with the Department of Mechanical Engineering, National Chiao Tung University (NCTU), Hsinchu, Taiwan. $\mathrm{He}$ received the Ph.D. degree in mechanical engineering from the University of California at Los Angeles, Los Angeles, CA, USA, in 2009 where he was a Post-Doctoral Researcher in 2009. From 2009 to 2011, he was with the MEMS Research and Development Department, Taiwan Semiconductor Manufacturing Company, Hsinchu. Since 2011, he has been at NCTU as a Faculty Member. His research interests are smart sensors, actuators, energy harvesters, MEMS, NEMS, and nanomagnetoelectrics. 


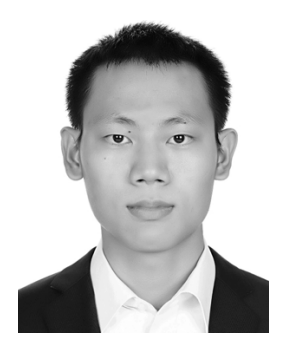

Tzu-Wei Liu received the B.S. degree in mechanical engineering from National Chiao Tung University, Hsinchu, Taiwan, in 2013, where he is currently pursuing the M.S. degree in mechanical engineering. His research interests include magnetomechanicalelectrical coupling devices, energy harvesters, and smart materials applications. His M.S. advisor is Professor T.-K. Chung.

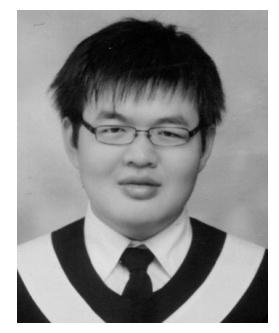

Hou-Jen Chu received the B.S. and M.S. degrees from the Department of Chemical and Materials Engineering, National Central University, Jhongli, Taiwan, and the Department of Mechanical Engineering, National Chiao Tung University, Hsinchu, Taiwan, in 2010 and 2013, respectively. His research interests include NEMS, magnetomechanical-electrical coupling devices, semiconductor manufacturing technology, and smart materials applications. His M.S. advisor was Professor T.-K. Chung.

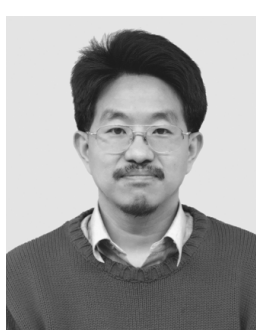

Wensyang Hsu received the M.S. and Ph.D. degrees from the Department of Mechanical Engineering, University of California at Berkeley, Berkeley, CA, USA, in 1990 and 1992, respectively. He is currently a Professor and the Chairman of the Department of Mechanical Engineering at National Chiao Tung University, Hsinchu, Taiwan. His current interests include MEMS technologies, wireless LC transducers, and biomedical devices.

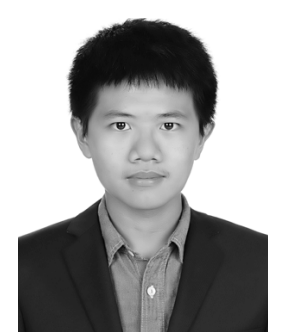

Po-Chen Yeh received the B.S. degree in mechanical engineering from National Chiao Tung University, Hsinchu, Taiwan, in 2013, where he is currently pursuing the Ph.D. degree at the Department of Mechanical Engineering. His research interests are nanomagnetoelectric coupling, smart materials, current sensors, MEMS, NEMS, and wireless sensing. His Ph.D. advisor is Professor T.-K. Chung.

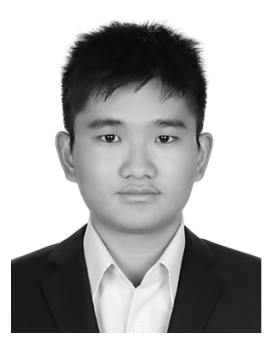

Chin-Chung Chen received the B.S. degree in mechanical engineering from National Chiao Tung University, Hsinchu, Taiwan, in 2013, where he is currently pursuing the Ph.D. degree in mechanical engineering. His research interests are applying magnetoelectric coupling in micro/nano-scale energy harvesters, NEMS, MEMS, and wireless sensing. His Ph.D. advisor is Professor T.-K. Chung.

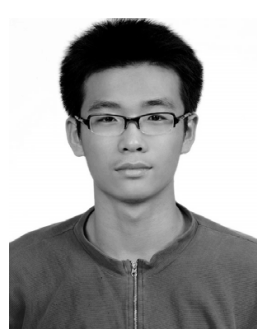

Meng-Shiue Lee received the B.S. and M.S. degrees in mechanical engineering from National Chung Cheng University, Chiayi City, Taiwan, and National Chiao Tung University, Hsinchu, Taiwan, in 2010 and 2012, respectively, where he is currently pursuing the Ph.D. degree in mechanical engineering. His research interests are MEMS technology, electromicrofluidics, and biomedical devices.

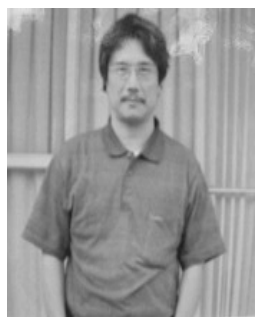

Yuh-Shyong Yang received the Ph.D. degree from the Department of Biochemistry, University of Wisconsin, Madison, WI, USA, in 1987. He is currently a Professor with the Department of Biological Science and Technology, National Chiao Tung University, Hsinchu, Taiwan. His current interests include enzyme, protein, engineering, proteomics, mechanisms of enzyme action and protein engineering, bioelectronics, and enzyme chip. 